magnetic field enhancements associated with surface plasmon resonances over the non-enhanced Raman scattering methods. However, SERS requires the target molecule to adsorb onto a metal surface to benefit from surface plasmon resonance. Consequently, it has been difficult to detect biological molecules that have a low affinity for metal surfaces, and only a few types of molecules have been detected by SERS with single-molecule sensitivity to date. The researchers said that huge signal gain can be obtained by combining SERS and coherent anti-Stokes Raman scattering (CARS) as is done in surfaceenhanced coherent anti-Stokes Raman scattering (SECARS). The researchers further improved the sensitivity of SECARS over the earlier reported work, and achieved single-molecule detection of some biomolecules not detected earlier using this method.

The researchers said that SECARS background originating from metal and water has limited the sensitivity of previous works. The research group used several methods, such as a polarizationsensitive CARS setup, minimized probe volume, and the use of aggregated colloidal silver nanoparticles instead of planar substrates, to reduce the background. The SECARS signals of biological molecules were increased by the addition of lithium chloride salts, which were found to be optimal for generating strong surface enhancements.

The researchers then demonstrated the power of SECARS by detecting two DNA nucleotides, deoxyadenosine monophosphate (dAMP) and deoxyguanosine monophosphate (dGMP), and a peptide-angiotensin-I peptide. Although dAMP has been detected at the single-molecule level by SERS, the SECARS signal was approximately 1000 times stronger than the SERS signal for dAMP at single-molecule concentrations. The SECARS signal of dGMP was at least 100 times stronger than the SERS signal of dGMP. The researchers believe that this is the first reported detection of dGMP at single-molecule concentrations.

The detection of the peptide shows that the SECARS technique can be applied to biological molecules other than nucleotides. According to the research group, the ultrasensitive detection of nonlabeled peptides and proteins has potential applications in pathogen detection, disease monitoring, and drug discovery.

VIVEK RANJAN

\section{Cell Membranes Integrated into CNT Devices}

Integrating biological systems and processes with nanofabricated structures is a critical challenge for nanotechnology. In the May issue of Nano Letters, J.-C. Gabriel, G. Grüner, and their co-workers reported on their work, performed at Nanomix Inc., on the integration of the cell membrane of Halobacterium salinarum with carbon nanotube (CNT) network transistors, wherein both the biological and nanoelectronic structures preserve their functionality and are able to electronically interact with each other.

The researchers fabricated nanotube network field-effect transistors, where the semiconducting channel is formed by a network of predominantly semiconducting nanotubes. A monolayer of the purple membrane from Halobacterium salinarum was then deposited on the exposed semiconductor channel. Evidence for the integration of the membrane with the device comes from changes in the device characteristics of the transistor.

The purple membrane contains bacteriorhodopsin, which has a permanent electric dipole moment pointing from the extracellular side of the membrane toward the cytoplasmic side. The researchers prepared three sets of devices. The first had the membrane dipole randomly oriented on the nanotubes, while the second and third sets had the cytoplasmic or extracellular side of the membrane facing the nanotubes. Prior to integration of the membrane, the nanotube devices by themselves worked as $p$-type transistors with good conduction at negative gate voltages and no conduction at positive gate voltages. The voltage at which the device turns on is known as the threshold voltage and is different depending on the sweep direction of the gate voltage (i.e., it is hysteretic). The hysteresis is known to be produced by water adsorbed on the substrate.

For the mixed-orientation nanobioelectronic device, deposition of the cell membrane resulted in a narrower hysteresis compared with the hysteresis of the nanotube devices alone. The researchers attributed this effect to desorption of water from the nanotube surface as the membrane displaced water. Also, the turn on for this device was less sharp, indicating a change in carrier mobility for the nanotube networks arising from random scattering potentials due to the electric dipoles of rhodopsin. The oriented membrane devices also showed a loss of hysteresis and a shift of the threshold voltage in different directions for the two different orientations of the membrane. Since the threshold voltage is correlated to the position of the Fermi level in nanotubes, the shift in the threshold voltage is indicative of a charge induced in the nanotube by the electric dipole of the membrane. The magnitudes of the shifts are different for the two orientations, implying asymmetry in the charge distribution of rhodopsin, with the dipole being closer to the cytoplasmic side.

Grüner, who is a professor at UCLA and advisor to the project, said that this work "opens up avenues for what could be called 'cellectronics,' the electronic detection and also modification of biological processes at the cellular level."

SARBAJIT BANERJEE

\section{Continuous-Wave $1.94 \mu \mathrm{m}$ Laser Based on $\mathrm{Tm}_{\mathrm{BaY}} \mathrm{B}_{2} \mathrm{~F}_{8}$ Lases from $1849 \mathrm{~nm}$ to $2059 \mathrm{~nm}$}

Efficient, widely tunable solid-state laser sources in the near-infrared region around $2 \mu \mathrm{m}$ have potential applications in remote sensing and gas detection, high-resolution spectroscopy, frequency metrology, and medicine. Thulium doping of crystals can be used in this wavelength region because of the ${ }^{3} \mathrm{~F}_{4}-{ }^{3} \mathrm{H}_{6}$ optical transition of $\mathrm{Tm}^{3+}$ ions that in most host media yields a very broad emission with a maximum at wavelengths of 1.8-1.9 $\mu \mathrm{m}$. In addition, $\mathrm{Tm}^{3+}$ has the advantage that it can be directly diodepumped at $\sim 785 \mathrm{~nm}$, and shows a quantum efficiency close to 2 (i.e., each pump quantum absorbed yields two Tm ions in the upper laser level).

In the April 15 issue of Optics Letters (p. 854), G. Galzerano and P. Laporta of Politecnico di Milano, in a joint collaboration with $\mathrm{M}$. Tonelli and colleagues from the Università di Pisa, Italy, described the growth, spectroscopic characterization, and laser action of a diode-pumped laser based on a Tm-doped fluoride crystal $\left(\mathrm{Tm}: \mathrm{BaY}_{2} \mathrm{~F}_{8}\right)$. They reported a roomtemperature, diode-pumped laser oscillator widely tunable over a $210 \mathrm{~nm}$ interval, from $1849 \mathrm{~nm}$ to $2059 \mathrm{~nm}$, with a maximum continuous-wave (cw) output power of $\sim 150 \mathrm{~mW}$ at $1920 \mathrm{~nm}$ by use of a $0.5 \%$ output coupler transmission.

The researchers said that fluoride crystals, despite thermomechanical properties that are usually poorer than those of most oxides, present some advantages in terms of laser performance. These include low phonon energy, longer fluorescence lifetime, lower upconversion losses, reduced thermal lensing, and extremely low-beam depolarization under strong pumping. The crystal was grown in a homemade Czochralski furnace, starting with $\mathrm{BaY}_{2} \mathrm{~F}_{8}$ powder and adding $\mathrm{BaF}_{2}$ and $\mathrm{TmF}_{2}$ powders. The best laser results were obtained for a Tm doping level of $12 \%$. Crystallinity and crystallographic orientation were determined from x-ray 